\title{
Diagnostics of Plasma Channel for HIF Transport *
}

\author{
D. M. Ponce ${ }^{a}$, C. Niemann ${ }^{c}$, S. S. Yu ${ }^{a}$, W. P. Leemans ${ }^{a}$, \\ T. J. Fessenden ${ }^{a}$, G. Dahlbacka ${ }^{a}$, W. M. Sharp ${ }^{\mathrm{b}}$, \\ A. Taushwitz ${ }^{a}$ \\ ${ }^{a}$ Lawrence Berkeley National Laboratory, Berkeley, CA. USA \\ ${ }^{\mathrm{b}}$ Lawrence Livermore National Laboratory, Livermore, CA. USA \\ ${ }^{\mathrm{c}}$ Gesellschaft für Schwerionenforschung, Darmstadt, Germany
}

\begin{abstract}
An alternate technique for heavy ion final transport, from the driver to the target, is by the use of the self-standing Z-pinched plasma channel. Experiments conducted at the Lawrence Berkeley National Laboratory have produced $40 \mathrm{~cm}$ long stable plasma channels with a peak discharge current of $55 \mathrm{kA}$ in a 7 torr nitrogen gas fill. These channels are produced using a double pulse discharge scheme, namely, a pre-pulse discharge and a main capacitor bank discharge. It is postulated that the channel's insensitivity to MHD instabilities within the time scale relevant to beam transport is due to the wall effect that the pre-pulse discharge creates. This is accomplished by leaving a gas density depression on the channel's axis after hydrodynamic expansion. Since the pre-pulse discharge creates the initial conditions for the main bank Z-pinch, it is critical to understand how to control and engineer the pre-pulse. Here we present some of the results of ongoing experiments geared to understand the underlying physics of the LBNL Z-pinch plasma channel. Schlieren and phase contrast measurements show the radial propagation of a shock wave during the pre-pulse discharge and suggest indirectly the evidence of the on axis gas density depression, that is believed to be $<1 / 10$ of the original gas fill pressure. For the main bank Z-pinch, interferometry show an integrated electron line density of $1.6 \times 10^{17} \mathrm{~cm}^{-2}$ for a $15 \mathrm{kV}$ discharge on axis. These measurements coupled with Faraday rotation measurements will indicate ultimately the current density distribution in the channel. This data will be used to benchmark simulation codes.
\end{abstract}

\footnotetext{
ऋ Work supported by the Director, Office of Energy Research [Office of Fusion Energy], U.S. Department of Energy under Contract No. DE-AC03-76SF00098.
} 


\section{Introduction}

The idea of using plasmas and plasma Z-pinches to transport charged particle beams has been investigated for some time now [1]. There is a renewed interest of this old concept since there are many practical applications of this idea. This variety of applications raises many questions that still remain unanswered. One application, the one discussed here, concerns the use of a plasma Z-pinch to transport a heavy ion beam for the production of inertial confinement fusion energy [2]. The channel will transport the beam from the final set of quadrupole magnets of the driver into the target chamber and deliver the beam to the fuel target. The immediate advantages that the channel offers are the relaxation of both the beam's emmitance and momentum spread constraints, thus simplifying the driver design [3]. The natural plasma's short space-charge neutralization time constant and the long magnetic diffusivity are the main reasons for this. Although we have these advantages, there are many obstacles to overcome as well. The Z-pinch is prone to the many MHD instability modes. Once one of these modes sets in, the channel would be ineffective on delivering the beam to the target. For a target chamber scenario where the standoff distance would be of approximately $6 \mathrm{~m}$, there are questions of premature voltage breakdown to the chamber walls. We believe that these two hurdles have been overcome. The LBNL plasma channel experiment has demonstrated $40 \mathrm{~cm}$ long stable Z-pinch channels with no premature breakdown to the chamber walls and with a peak current of 55kA [4]. This is accomplished with a double discharge technique. This paper looks into understanding and explaining the relevant physics behind the stability.

\section{The experimental setup and diagnostics}

The LBNL plasma channel experiment schematic is shown on Figure 1. It consists of a chamber with two copper electrodes separated by a $40 \mathrm{~cm}$ distance. The channel formation is done in a three step sequence: i) laser pre-ionization; ii) pre-pulse capacitor discharge; iii) main bank capacitor discharge. The chamber is filled with 6 to 7 torr of nitrogen and 500mtorr to 1torr of benzene. Benzene is used due to its relatively high UV absorption cross section to produce initial seed electrons to aid the discharge. This particular version of the experiment uses a frequency quadrupled $(\lambda=266 \mathrm{~nm}) \mathrm{Nd}$ :YAG laser with a $100 \mathrm{~mJ}, 10 \mathrm{~ns}$ pulse width. The laser beam is passed through the experimental chamber along the preferred channel axis. The frequency doubled component of the laser $(\lambda=532 \mathrm{~nm})$ is used to trigger the pre-pulse capacitor via a brass ball pair spark gap. The $150 \mathrm{nF}$ pre-pulse capacitor is previously charge to $7 \mathrm{kV}$. Upon its triggering, there is a 5 to $15 \mu$ s period where the seed electrons get accelerated under the applied electric field causing joule heating on the 
defined path-length. Once the proper ratio of electric field to pressure are met, electron avalanching occurs, thus initiating the pre-pulse channel electric breakdown. During breakdown, the electric energy from the pre-pulse capacitor is dumped in about a microsecond producing a radial shock wave. After this shock wave, a deep long lived gas density depression, estimated through simulations at $<1 / 10$ of $P_{0}$ is left behind to serve as the preferred electric breakdown path for the main bank capacitor discharge, which is fired 30 to $40 \mu$ s after the pre-pulse breakdown occurs. The main bank trigger time can be precisely set to within a nanosecond. The main capacitor bank in our experiment is charged from $15 \mathrm{kV}$ to $30 \mathrm{kV}$. With this technique, the pre-pulse sets the initial conditions for the main bank discharge by eliminating the premature electric breakdown to the chamber walls and producing a high pressure wall that helps MHD stabilize the main bank discharge. In a full scale target chamber where benzene could not be used, seed electrons can be easily created with a chirped pulsed amplified Ti:Sapphire femtosecond laser.

Several diagnostics were used to characterize both the pre-pulse and the main bank discharges. For the pre-pulse, a schlieren and zernike phase contrast setup was used to measure the hydrodynamics of the neutral gas during the shock wave expansion. The phase contrast diagnostics setup is shown on figure 2. The difference between the schlieren and the phase contrast techniques is the replacement of the typical schlieren "knife edge" with a "phase mirror". This makes the intensity variations on the channel's image be proportional to the gas density and enhances sensitivity [5][6]. For the main bank discharge, a Michelson interferometer, diagram shown on figure 3, was used to measure the plasma electron density. The probing light source for both the schlieren and interferometer setups was a pulsed Nd:YAG laser at $1.064 \mu \mathrm{m}$ with a $20 \mathrm{~ns}$ pulse width which gave the necessary time resolution. For the phase contrast measurements, the $\lambda=515 \mathrm{~nm}$ line of a $\mathrm{cw} \mathrm{Ar}{ }^{+}$laser together with a streak camera were used. All images were acquired through an eight bit COHU CCD camera, frame grabber board, and computer system.

\section{Experimental Data and Analysis}

Schlieren and phase contrast image data show the pre-pulse shock wave radial position as a function of time. This is shown on figure 4. From the schlieren images we can see the shock propagating outward delimiting the central region of lower gas density. From the phase contrast streak images we see the time evolution of the pre-pulse. One possible way of interpreting this data, and as a first approximation, is by using a blast wave solution[7][8]. According to the blast wave theory, the radius of the shock $r_{\text {shock }}$ scales like:

$$
r_{\text {shock }}(t)=\xi_{0}\left(E t^{2} / \rho\right)^{1 / 5}
$$


where $\xi_{0}$ is a number of the order of unity, $E$ is the energy deposited in the blast, and $\rho$ is the undisturbed density. It is not clear if radiative losses are large enough to be considered, although a snow-plow model is not out of the question. The data is not best fitted to a wave blast model directly, although best fit occurs for a modified model where $r_{\text {shock }}(t) \alpha t^{2 / 3}$. Figure 5 show plots where the experimental data has been fitted to the $r_{\text {shock }}(t) \alpha t^{2 / 3}$ empirical model. Also shown in figure 5 is the shock wave expansion rate as a function of time calculated from this model. A comparison of the shock evolution with the output of CYCLOPS, our work in progress simulation code, is shown also on figure 4. CYCLOPS is a 1-D Lagrangian code that incorporates a hydrodynamic model with an ideal-gas closure. Maxwell's equations are coded to be solve self consistently for the azimuthal magnetic field and the longitudinal electric field. It also incorporates a lumped circuit equation that permits the input of the applied voltage and laser spot size as initial conditions. Electrical conductivity is calculated via an electron density rate equation that incorporates both avalanching and recombination models. A more detailed description of the code will be presented in the future.

Following the blast wave solution, it is estimated that about $1 \%$ of the prepulse capacitor energy goes into the pre-pulse hydrodynamic expansion. Runs were made for different values of pressure, applied voltage, and gas species. Figure 6 shows this data. A theoretical model explaining the stabilizing effect of a background surrounding gas in a z-pinch has been proposed by Manheimer et al. [9]. They show that the inertial effects of a gas density gradient is to reduce the MHD instability growth rate by a factor of $\left(\rho_{p} / \rho_{g}\right)^{1 / 2}$, where $\rho_{p}$ is the density of the pinch and $\rho_{g}$ is the density of the surrounding gas. Explicitly, the growth rate $\Gamma$ is given by:

$$
\Gamma \approx k v_{A p}\left\{\left(\frac{\rho_{p}}{\rho_{g}}\right)^{1 / 2}\left[1+k r_{p}\left(\frac{P_{p}}{P_{g}}\right)^{1 / 2}\right]\right\}
$$

where the subscripts $p$ indicating pinch parameters, $g$ gas parameters, $k$ is the instability mode wavenumber, $v_{A p}^{2} \equiv B_{0} / \mu_{0} \rho_{p}, r_{p}$ the pinch radius and $P$ the pressure. The growth rate for a pinch in vacuum is:

$$
\Gamma \approx k v_{A p}\left[-\ln \left(\frac{1}{2} k r_{p}\right)-\gamma_{E}\right]
$$

where $\gamma_{E}=0.5772 \cdots$ is Euler's constant. Manheimer further does a case analysis for lightning where the discharge conditions are quite similar to the discharges described in this experiment. They show that for the density ratio $\rho_{g} / \rho_{p}>>1$ the growth rate can be smaller by at least an order of magnitude putting the growth rate for our discharges on the order of $10^{-3} \mathrm{~s}$ with $k r_{p}<1$ and $m=1$ mode instability (conditions that present the largest growth rates.) 
Interferometry measurements yield the plasma electron density for the main bank discharge. The fractional plasma fringe shift at different times is shown in figure 7 . For the Michelson interferometer, the plasma density is given by equation 4:

$$
N_{e}=\frac{\pi\left(1-\eta^{2}(r)\right)}{\lambda^{2} r_{\mathrm{e}}}
$$

with

$$
\eta(r)=\eta_{0}-\frac{\lambda}{4 \pi} \int_{y}^{\infty} \frac{\frac{d}{d y} K(y)}{\sqrt{y^{2}-r^{2}}} d y
$$

where $N_{e}$ is the electron density, $\lambda$ the wavelength of the probing light, $r_{\mathrm{e}}$ the classical electron radius, and $\eta(r)$ the index of refraction of the electrons in the plasma. The raw data fringe shift information, which generates the function $K(y)$, was fitted to a polynomial and abel inverted (equation 5) giving the desired electron index of refraction function. Typical peak electron densities on axis are of the order of $3.5 \times 10^{16} \mathrm{~cm}^{-3}$ for a 5 torr $\mathrm{N}_{2}$ gas fill, $15 \mathrm{kV}$ discharge. A couple of fringe pictures are shown on figure 8. Effect of neutrals on the fringe shift is negligible for the path-length the probe beam transverses $(\sim 2 \mathrm{~cm})$ and the probing laser beam wavelength. The occurrence of the Zpinch sits in the vicinity of $2 \mu \mathrm{s}$ in accord with previous measurements [4]. An encouraging feature emerge from this data. When the pre-pulse channel fires properly, the main bank discharge shows no evidence of MHD instabilities for more than $10 \mu \mathrm{s}$. Together with the electron density distribution, the current density distribution, $J$, needs to be measured. Uniformity of $J$ within the pinch is important for beam transport. This latter part needs to be investigated and this is where our efforts are focused presently [10].

\section{Conclusion}

These results will be used to benchmark simulation codes to further predict the plasma channel's behavior in the real target chamber. Presently, a one dimensional fluid code, CYCLOPS, is being developed and used to model the LBNL plasma channel. This code will incorporate avalanche ionization and recombination, hydrodynamics, and a realistic representation of the external circuit. More quantitative measurements of the channel are in order. The determination of the current density inside the Z-pinch will be revealed by the channel's magnetic field measurements [10]. Up to now, there are no definite objections to use a Z-pinch plasma channel approach to deliver a high intensity ion beam to the fusion target. After complete understanding of the 
channel's dynamics is achieved, full scale experiments will need to follow, as well as theoretical analysis and experiments to investigate beam-channel interactions. These experiments will require real high intensity ion beams to test these interactions with the plasma channel.

\section{References}

[1] D. Hinshelwood, J. Appl. Phys. 72, 4581 (1992).

[2] A. Tauschwitz, S. S. Yu, S. Eylon, R. O. Bangerter, W. Leemans, C. Peters, J. O. Rasmussen, L. Reginato, J. J. Barnard, W. M. Sharp, Fus. Eng. Des. 32-33, 493 (1996).

[3] S. S. Yu, et al, LBNL report 40669.

[4] T. J. Fessenden, M. C. Vella, HIFAR report 474.

[5] M. Born, E. Wolf, Principles of Optics (Pergamon Press, London, England, 1959).

[6] H. M. Presby, D. Finkelstein, Rev. Sci. Inst., 38, 1563, (1967).

[7] F. H. Shu, The Physics of Astrophysics, Vol. II, (University Science Books, Mill Valley, CA, 1992).

[8] L. D. Landau, E. M. Lifshitz, Fluid Mechanics (Pergamon Press, London, England, 1959).

[9] W. M. Manheimer, M. Lampe, J. P. Boris, Phys. Fluids 16, 7, 1126 (1973).

[10] C. Niemann, et.al:this conference. 


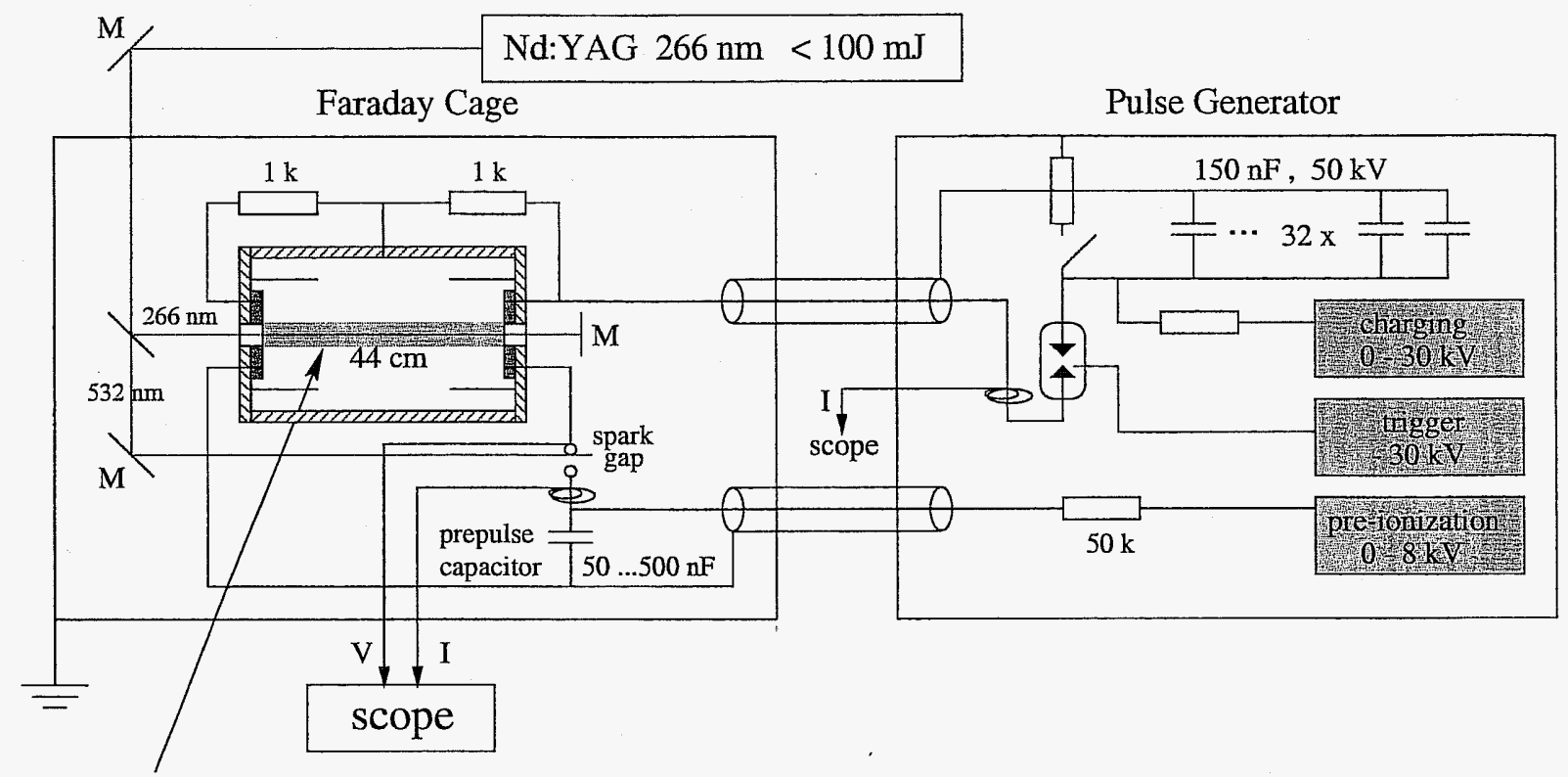

Plasma Channel

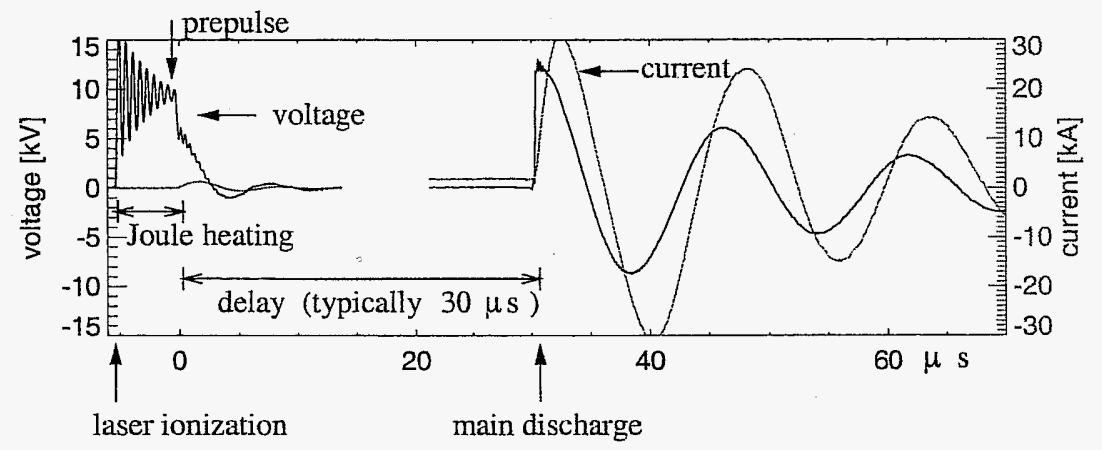

Fig. 1. LBNL Plasma Channel Experiment Schematic: Basic schematic of experimental setup, and phenomenology of pre-pulse and main bank discharges 


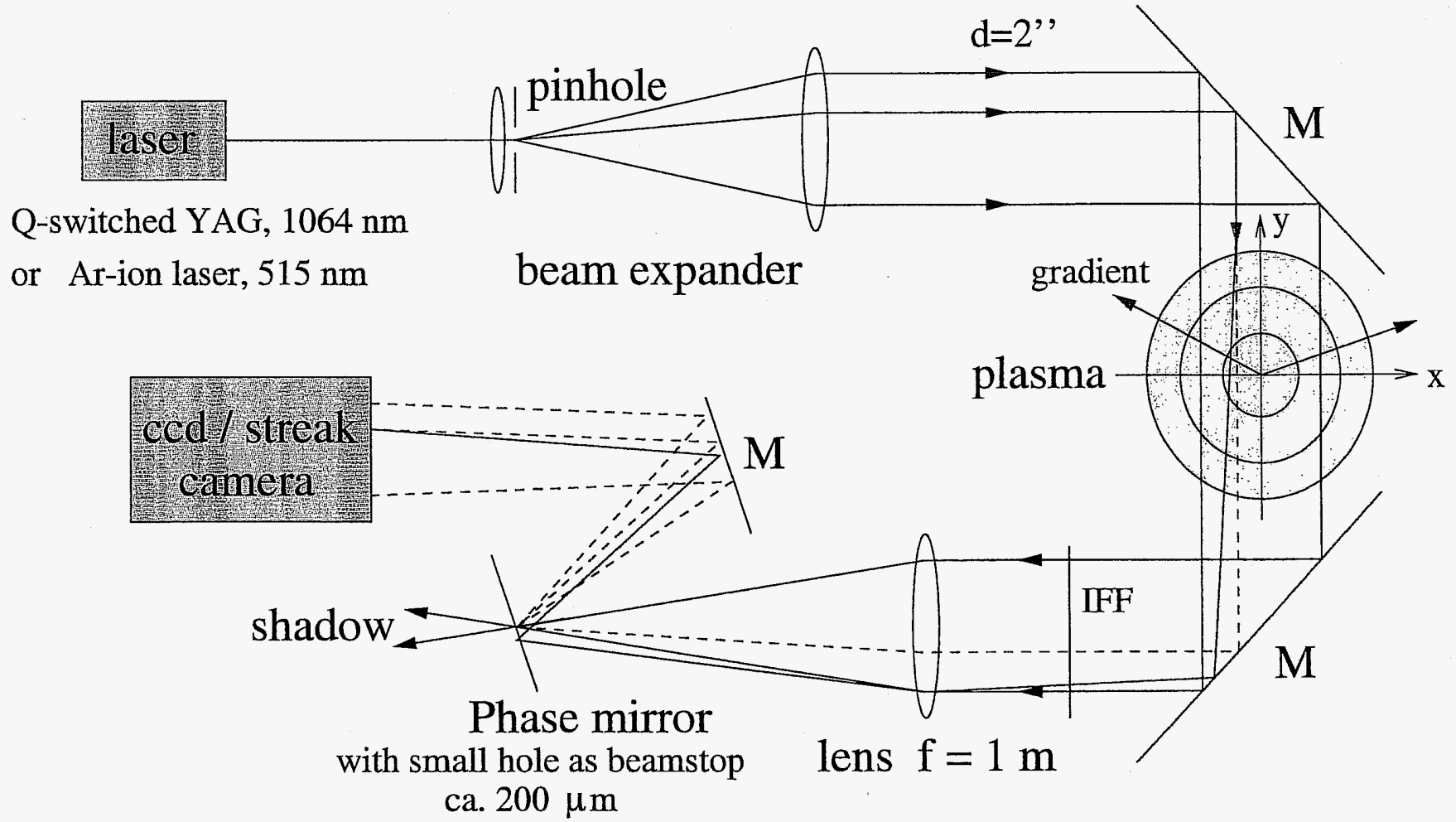

Fig. 2. Diagnostics: Zernike's Phase Contrast. $M=$ mirror, IFF = Interference filter.

For schlieren setup, one replaces the phase mirror by a knife edge 


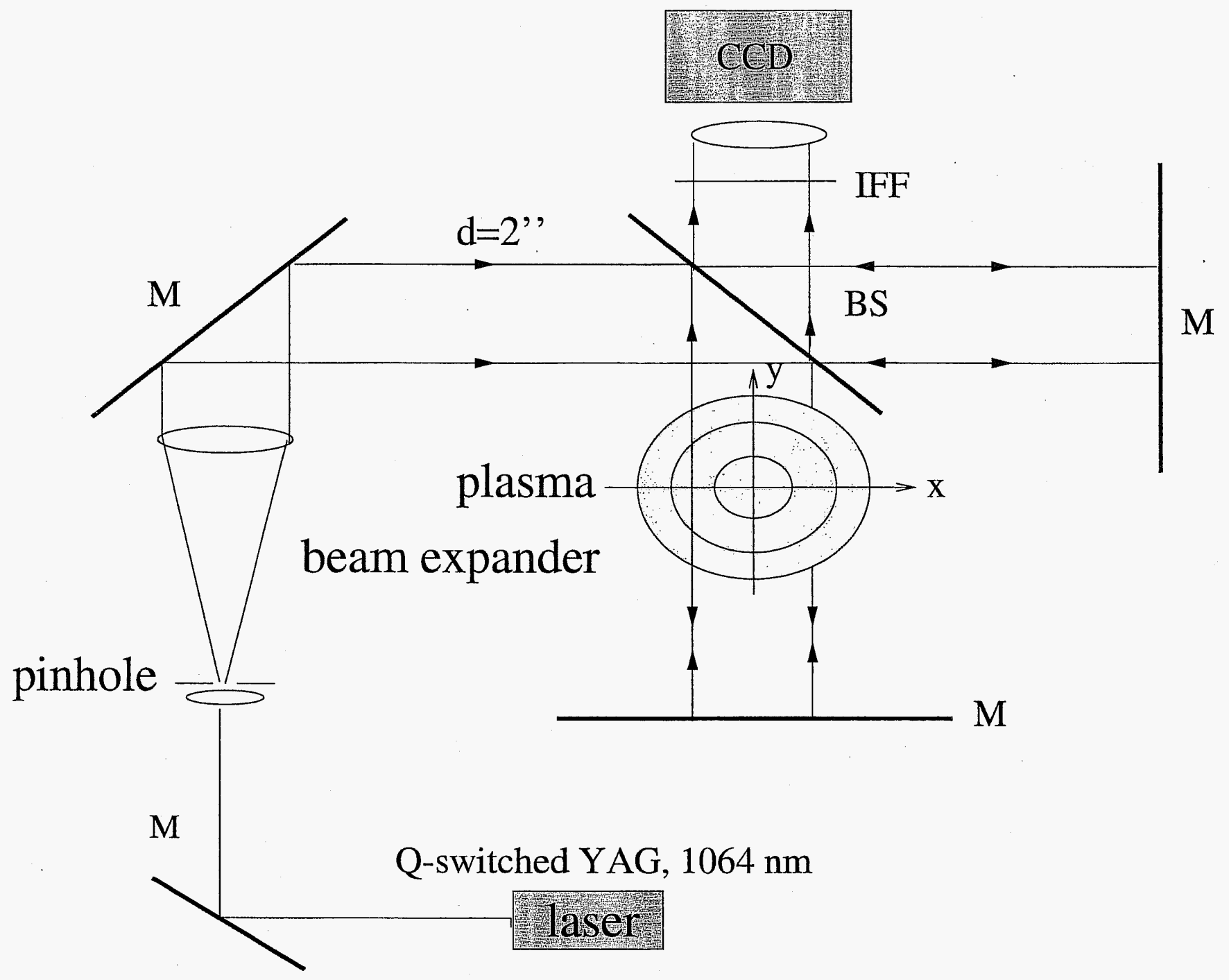

Fig. 3. Diagnostics: Michelson type interferometer. $\mathrm{M}=$ mirror, $\mathrm{IFF}=$ interference filter 

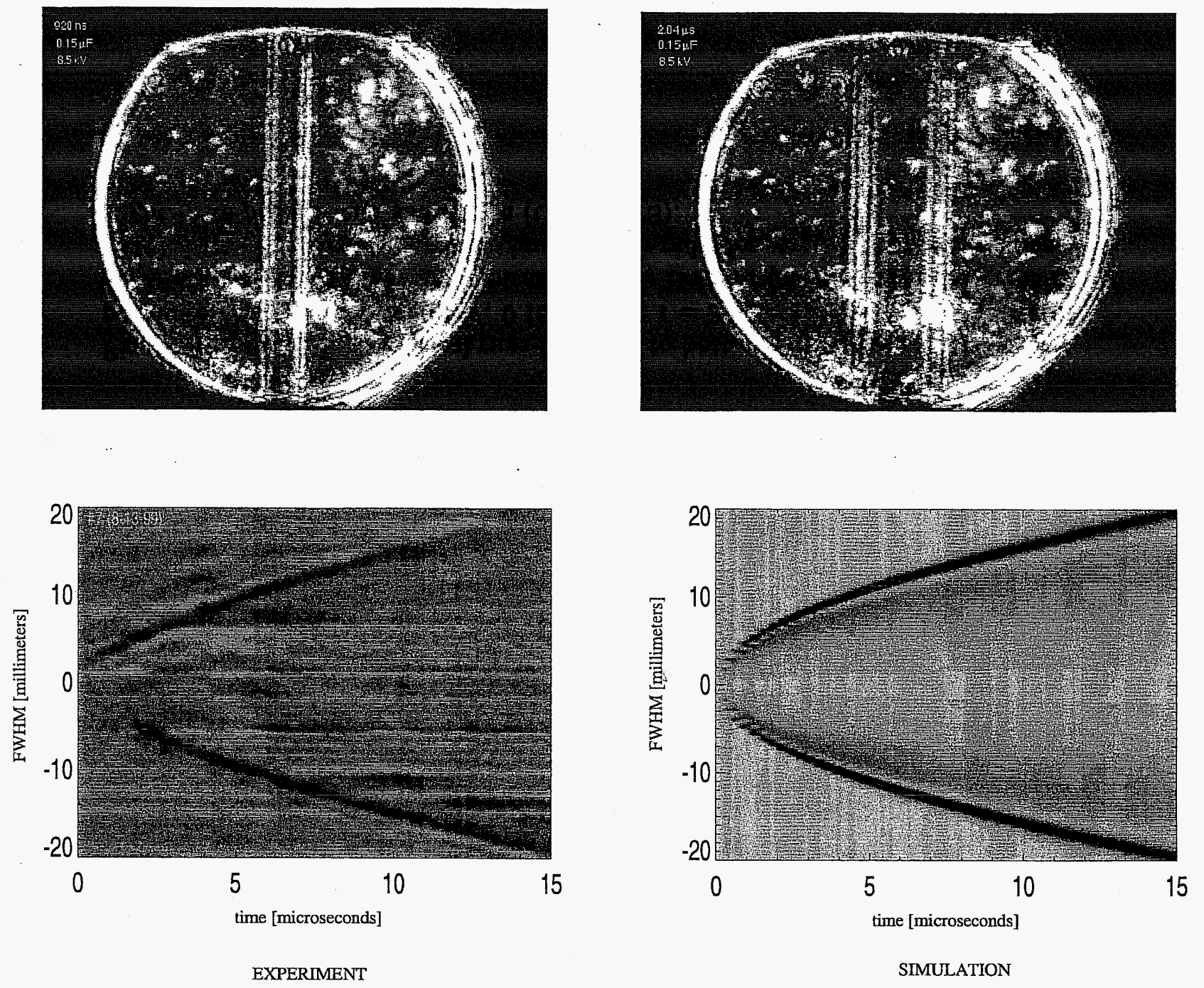

Fig. 4. Raw data: Top (channel runs from top to bottom).- Schlieren images of the pre-pulse $P=7$ torr, $V=8.5 \mathrm{kV}, 0.15 \mu \mathrm{F}$. Left $t=920 \mathrm{~ns}$; right $t=2.04 \mu \mathrm{s}$. Bright spots are carbon deposits from burnt benzene. Bottom (channel runs from left to right). Left.- Phase contrast streak image of pre-pulse for same discharge conditions. Right.- Pre-pulse streak simulation (CYCLOPS). 

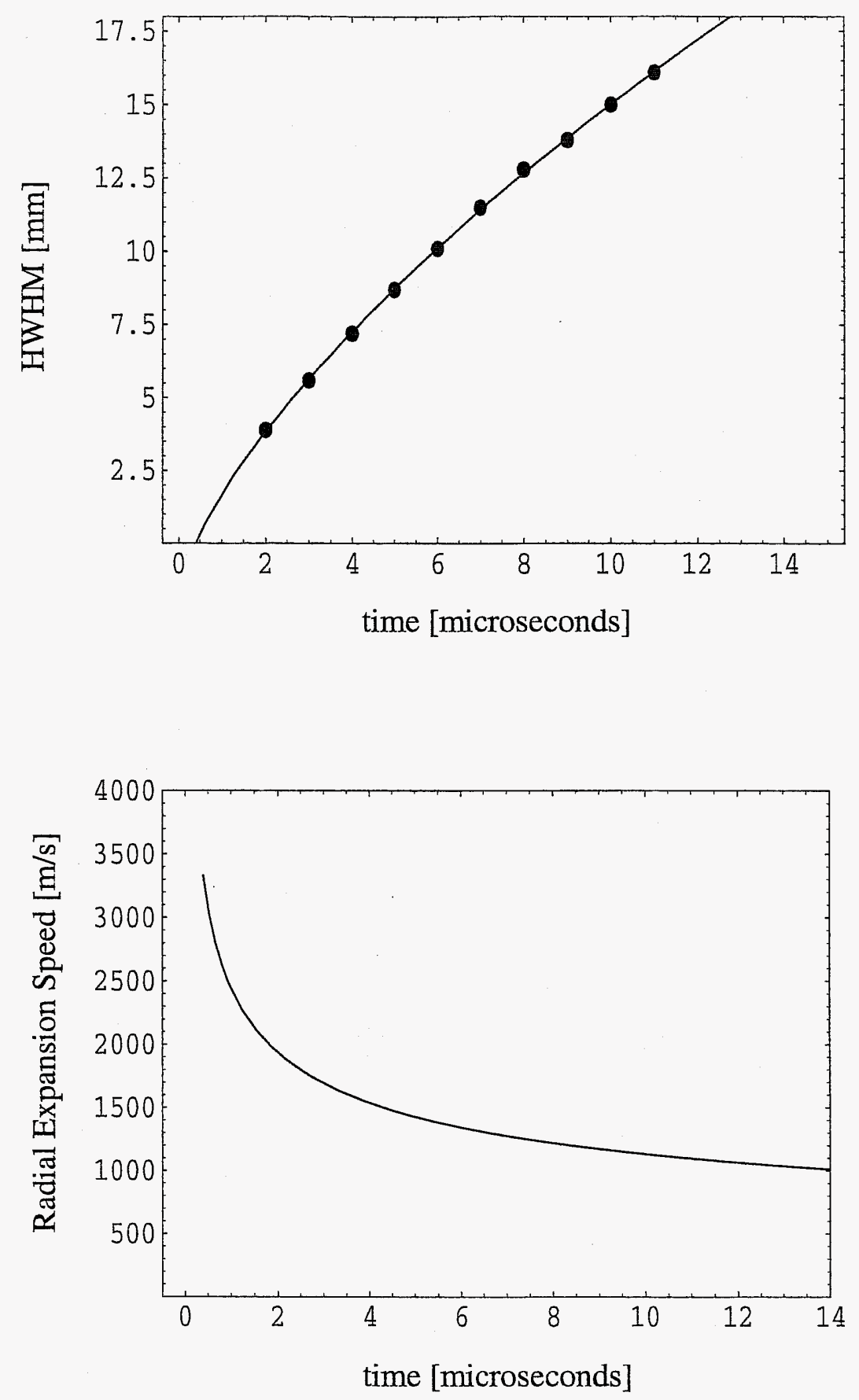

Fig. 5. Pre-pulse shock wave data: Top plot shows the empirical $2 / 3$ power fit to the experimental data. Bottom plot shows pre-pulse shock wave expansion rate as a function of time. Pre-pulse discharge parameters: 7 torr nitrogen, $7 \mathrm{kV}$. 


\section{DIFFERENT GAS SPECIES}
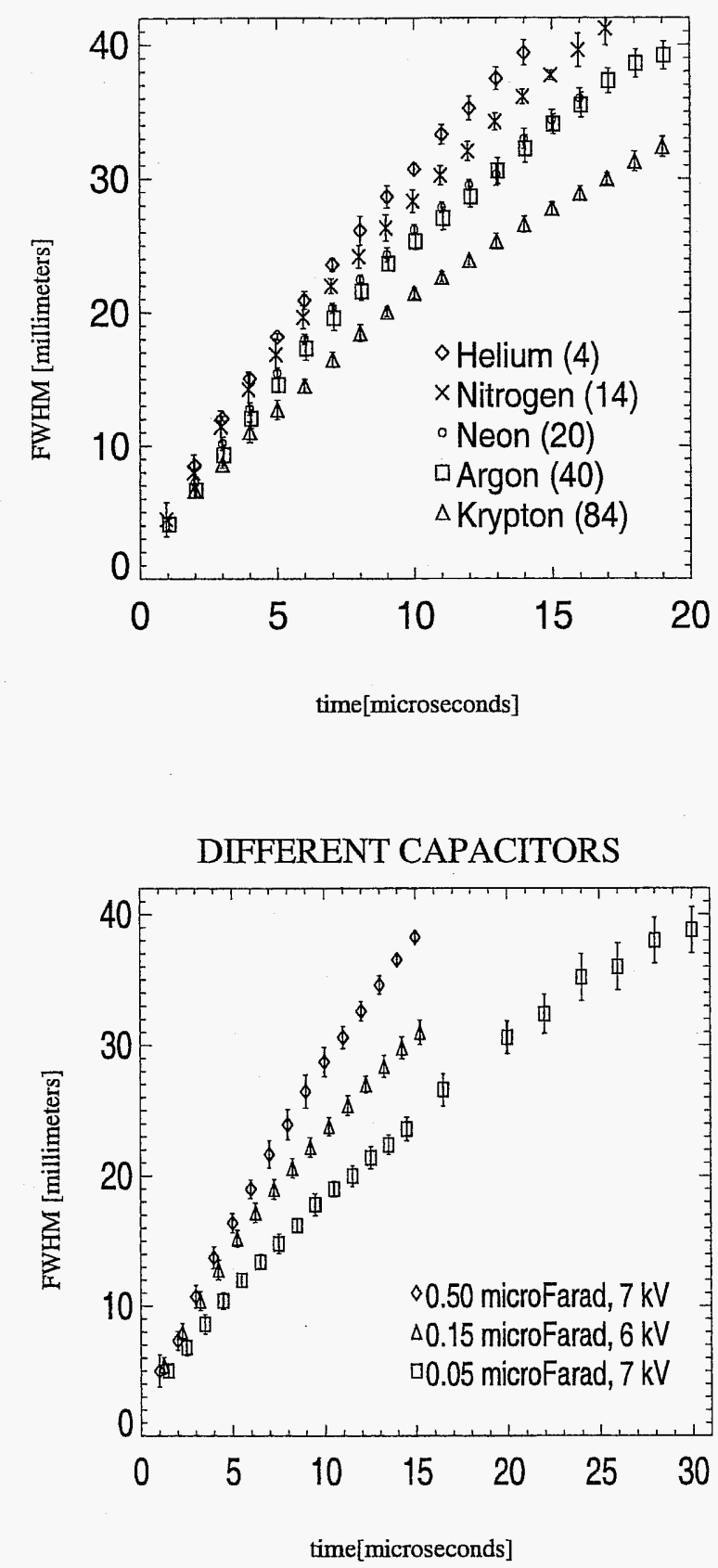

Fig. 6. Schlieren and phase contrast data. Plots show different runs for different conditions. Top shows the shock expansion as a function of gas species, 7 torr, $0.5 \mu \mathrm{F}$, $7 \mathrm{kV}$. Bottom shows pre-pulse shock wave expansion rate as a function of capacitor energy, 7torr nitrogen. 


\section{Fringe Shift, 5torr N2, 15kV, 0.15 microFarad}

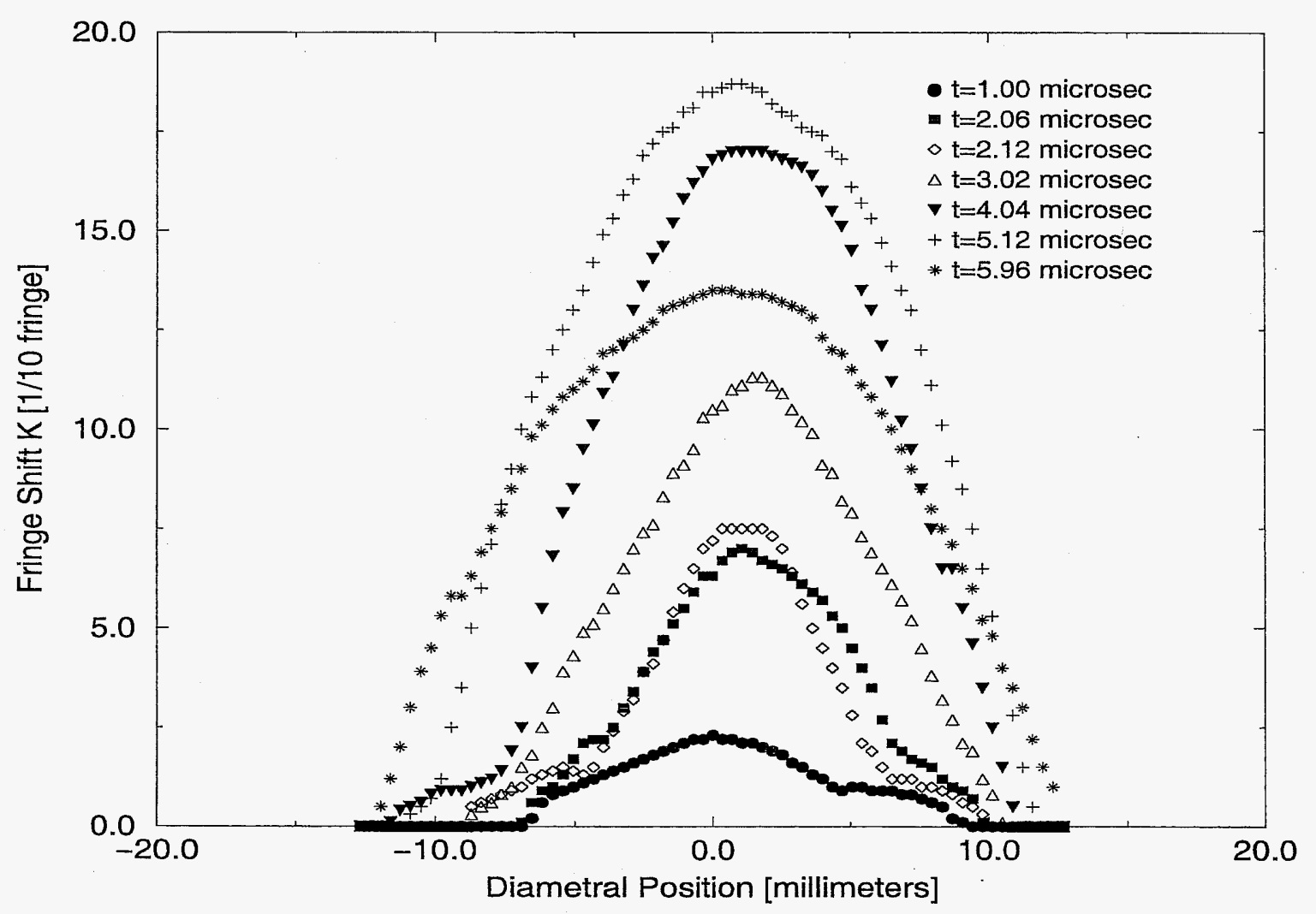

Fig. 7. Main bank discharge interferometric fringe shift: This data was fitted to an $8^{\text {th }}$ order polynomial, and abel inverted. Typical peak electron density on axis is $3.5 \times 10^{16} \mathrm{~cm}^{-3}$ 
$\mathrm{t}=2.96$ microseconds
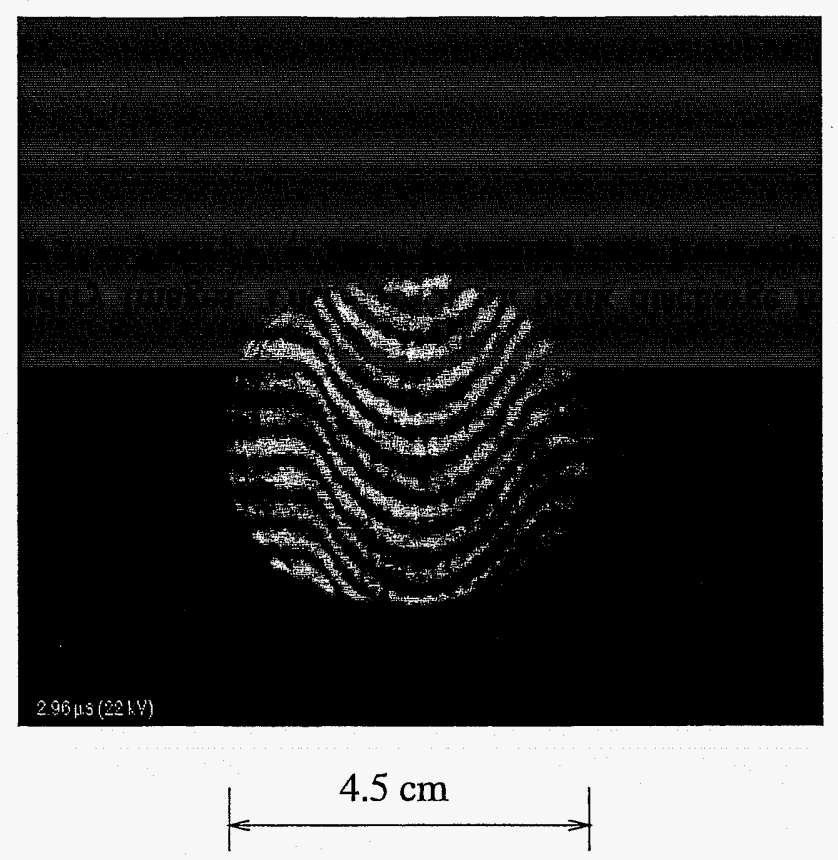

$\mathrm{t}=3.68$ microseconds

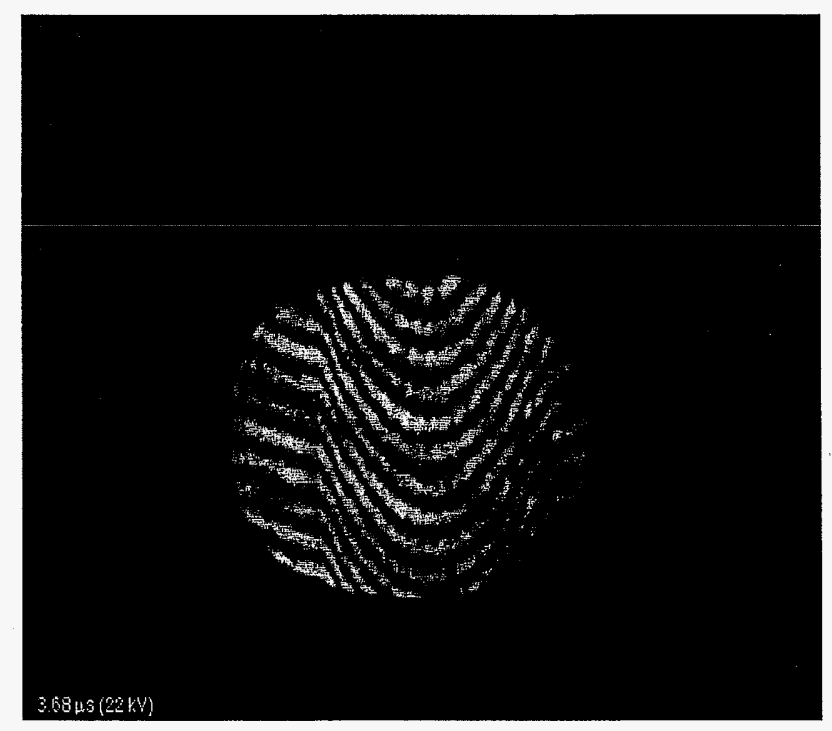

Fig. 8. Interferometry Images: These are main bank discharge fringe images. Discharge parameters $P=7$ torr, $V=22 \mathrm{kV}$ (Channel runs from top to bottom.) 